\title{
Multiexciton Absorption Cross Sections of CdSe Nanocrystals at Band-Edge Energy
}

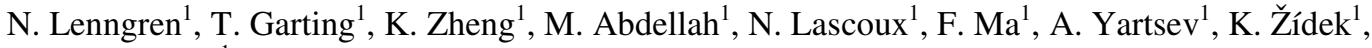 \\ and T. Pullerits ${ }^{1}$ \\ ${ }^{1}$ Department of Chemical Physics, Lund University, Box 124, 22100 Lund, Sweden
}

\begin{abstract}
Picosecond transient absorption signals of two kinds of cadmium selenide quantum dots were measured at various excitation intensities. The average number of excitons per quantum dot was calculated from a Poisson model, which together with kinetic parameters was used to determine exciton population kinetics. Exciton and multiexciton absorption cross sections were determined and analyzed in terms of the electronic states of the quantum dots.
\end{abstract}

\section{Background}

Semiconductor nanocrystals, called quantum dots (QDs), are attracting broad scientific attention both from fundamental and applied research. The interest is related to the intriguing opto-electronic properties of QDs, such as quantized energy levels and size-tunable bandgap. The effects are due to quantum confinement, occurring if the size of the QD is similar or smaller than the exciton Bohr radius.

In the context of solar energy, the potential of QDs in breaking the Shockley-Queisser thermodynamic limit via multiple exciton generation (MEG) is particularly attractive. However, the reported yields of the process are contradictory, and understanding of the basic principles is still obscure. The process is commonly described in terms of impact ionization where multiple excitons are generated in a sequential process. The observed dynamics are very fast - within the time resolution of the experiments carried out so far (50 fs) multiple excitons are formed.

MEG yields are usually determined from the relative amplitude of the characteristic fast multiexciton component of the band edge photoemission or photobleaching dynamics. For quantitative analyses one needs to have a good knowledge of the oscillator strengths of the transitions which contribute to the signal. As a preparation for quantitative analysis of MEG yields, we analyze transient absorption at the band edge to determine absorption cross sections for multiple excitons.

\section{Experimental}

Two kinds of QDs were used: CdSe QDs with diameter $5 \mathrm{~nm}$ (Lumidot, Sigma-Aldrich, called core QDs in the following) and core-shell (CdSe)ZnS QDs with core diameter $3 \mathrm{~nm}$ (synthesized by us, called core-shell QDs in the following). The pump pulse intensity, denoted in the text as factors of a reference intensity $I_{0}$, was varied by using neutral density filters. After the pump pulse, a probe pulse interacted with the sample providing transient absorption (TA) traces on a subpicosecond to

This is an Open Access article distributed under the terms of the Creative Commons Attribution License 2.0, which permits unrestricted use, distribution, and reproduction in any medium, provided the original work is properly cited. 
nanosecond timescale. The core QDs were probed by white light continuum in order to analyze the TA spectra. The core-shell QDs were probed by $526 \mathrm{~nm}$ and $575 \mathrm{~nm}$ light.

\section{Results and discussion}

The core QD TA spectra had negligible changes after $1 \mathrm{ps}$; therefore we use single-wavelength probes for the other samples. The bleach increases for higher excitation intensities, which is attributed to creation of multiple excitons via a sequence of absorptions. One can identify parts of the kinetics corresponding to different numbers of excitons present in the system, i.e. Auger relaxation of the multiexcitons [1], with fast multiple exciton decay being present for the highest intensities at $t<6 \mathrm{~ns}$, except at the lowest intensities. Results, normalized at long times, are shown in Figure 1.

\subsection{Calculation of ground-state absorption cross section}

Starting from Poissonian distribution of multiple exciton populations [2], we describe the normalized TA signal $\triangle O D / \triangle O D_{0}$ at long times $(t>6 \mathrm{~ns})$, where we only have one exciton per excited QD independent of excitation intensity, by an equation including the average number of excitons per quantum dot at $I_{0}$ as a parameter $\lambda_{0}$. From $\lambda_{0}$ we can also obtain the absorption cross section $\sigma_{a b s}$ at the pump wavelength.

Experimental noise and a possible minor contribution from the multiple excitons cause a spread in $\triangle O D / \triangle O D_{0}$ for different time points. We fit to the highest and lowest values of $\triangle O D / \triangle O D_{0}$ for all samples.

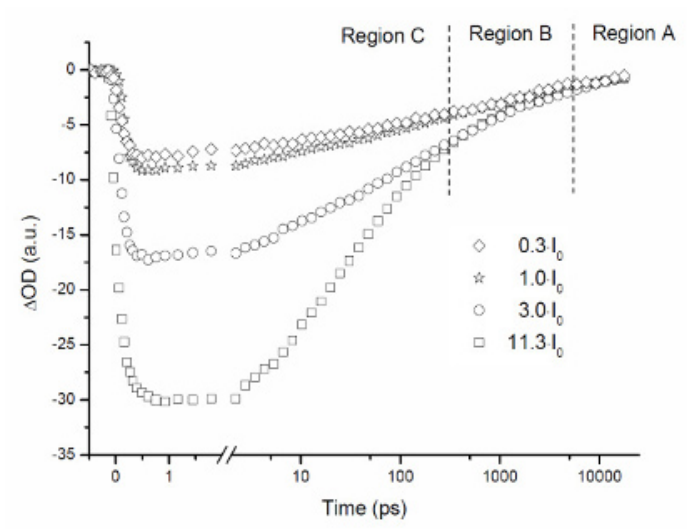

Fig. 1. TA kinetics of $5 \mathrm{~nm}$ QDs at $592 \mathrm{~nm}$ for the four different pump intensities, normalized at times longer than 6 ns. Faster kinetics from multiexcitons can be clearly identified for early times. In Region $\mathrm{C}$, three or more excitons are present, and in Region B, two excitons remain. All curves overlap at times over 6 ns (Region A), corresponding to the one-exciton signal.

\subsection{Kinetic model}

The TA signal $S_{n}(t)$ due to the $n$-exciton population $P_{n}(t)$ is obtained as

$$
S_{n}(t)=\left(A_{n, n+1}-E_{n, n-1}-A_{01}\right) \cdot P_{n}(t)=K_{n} \cdot P_{n}(t)
$$

where $A_{n, n+1}$ is absorption from the level $n$ to the level $n+1, E_{n, n-1}$ is stimulated emission from the level $n$ to the level $n-1$ and $A_{01}$ is the ground-state bleach (the optical density at the analysis wavelength). We extract $P_{n}(0)$ from the fits by using the Poissonian distribution and describe $P_{n}(t)$ 
by Pauli master equations where we only allow downwards population transfer with rate constants $k_{i}$. The rate constants are obtained by fitting sums of exponentials to the normalized traces.

By calculating the $K_{n}$ values for each intensity from both the lower and upper boundaries of $\lambda$ and their averages, and comparing them to the $\triangle O D$ signal, we find that $K_{n}$ coefficients obtained from the averaged $\lambda$ give a more accurate and self-consistent description of the system, which means that they will be used in the following.

\subsection{Calculation of absorption cross sections for $n$-excitons}

Returning to Eq. 1, we set up a system of $K_{n}$ equations. As the lowest level is a dark state [3, 4], we calculate $E_{10}$ from the radiative lifetime obtained from the long component of the kinetics together with the quantum yield, obtaining $E_{10} \approx 0.02 \cdot A_{01}$. Using relative multiexciton emission strengths from literature data [5] and extrapolation we obtain $A_{n, n+1}$ for $n=1$ to 4 and recalculate these to absorption cross sections.

Plots of absorption cross sections versus transition $(n$ to $n+1)$ are shown for all samples in Figure 2. At 2, 3, and 4 excitons, values from different intensities diverge, increasing the uncertainty. The trend for core-shell QDs probed at $575 \mathrm{~nm}$ and core QDs can be qualitatively explained via electronic structure of the band-edge transitions. The reduction of the absorbance at band edge occurs after filling the two $1 S_{e}$ states by electrons. When all 4 hole states of $1 S_{3 / 2}$ become filled, the absorption at the band-edge energy diminishes drastically. When core-shell QDs are probed at $526 \mathrm{~nm}$, several bands are involved, complicating the picture.
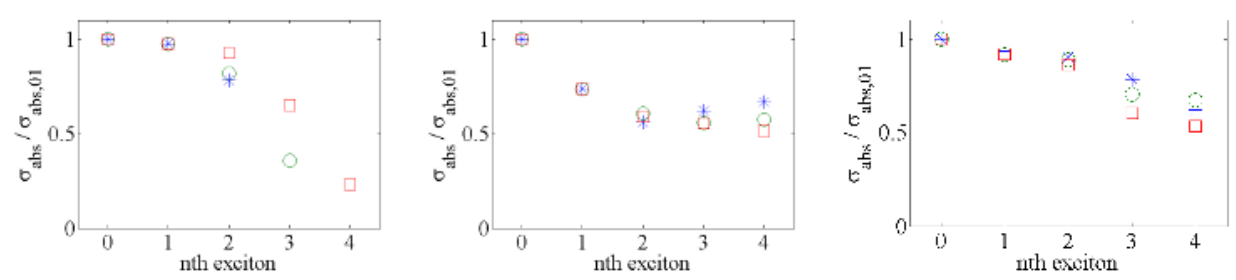

Fig. 2. Normalized absorption cross sections for the transition from $n$ to $n+1$ excitons, calculated at different intensities for core QDs (left), core-shell QDs probed at $526 \mathrm{~nm}$ (middle) and core-shell QDs probed at $575 \mathrm{~nm}$ (right). The absolute absorption cross section for the $0 \rightarrow 1$ transition is $19.0 \AA^{2}$ for core QDs, $2.60 \AA^{2}$ for core-shell QDs probed at $526 \mathrm{~nm}$ and $4.52 \AA^{2}$ for core-shell QDs probed at $575 \mathrm{~nm}$. Squares represent the highest intensity, circles the second-highest and stars the third-highest.

In conclusion, we measure picosecond transient absorption of CdSe quantum dots and use the results to determine absorption cross sections for multiexciton formation. Our method includes a self-consistency check to increase accuracy. These results are crucial to our ongoing MEG measurements. Based on the careful analysis of Auger relaxation of multiexcitons performed here, which avoids many of the assumptions that are usually made; we can determine MEG yields with high accuracy, hopefully resolving the present controversy.

\section{References}

1. V.I. Klimov, A.A. Mikhailovsky, D.W. McBranch, C.A. Leatherdale, M.G. Bawendi, Science 287, 1011 (2000).

2. M. Ji, S. Park, S.T. Connor, T. Mokari, Y. Cui, K.J. Gaffney, Nano Lett. 9, 1217 (2009).

3. M.G. Bawendi, P.J. Carroll, W.L. Wilson, L.E. Brus, J. Chem. Phys. 96, 946 (1992).

4. A. Efros, M. Rosen, M. Kuno, M. Nirmal, D. Norris, M. Bawendi, Phys. Rev. B 54, 4843 (1996).

5. B. Fisher, J.M. Caruge, D. Zehnder, M. Bawendi, Phys. Rev. Lett. 94, 087403 (2005). 